submitted to ApJ Letters 2000 Oct 21, revised 2000 Nov 28

\title{
Discovery of the Bright Trans-Neptunian Object 2000 EB173
}

\author{
Ignacio Ferrin ${ }^{1}$, D. Rabinowitz ${ }^{2}$, B. Schaefer ${ }^{2,3}$, J. Snyder ${ }^{2,3}$, N. Ellman ${ }^{2}$, B. Vicente ${ }^{4,8}$, A. \\ Rengstorf ${ }^{5}$, D. Depoy ${ }^{6}$, S. Salim ${ }^{6}$, P. Andrews ${ }^{2}$, C. Bailyn ${ }^{3}$, C. Baltay ${ }^{2}$, C. Briceno ${ }^{2,4}$, P. \\ Coppi $^{2,3}$, M. Deng ${ }^{2}$, W. Emmet ${ }^{2}$, A. Oemler ${ }^{7}$, C. Sabbey ${ }^{9}$, J. Shin ${ }^{2}$, S. Sofia ${ }^{3}$, W. van Altena \\ ${ }^{3}$, K. Vivas ${ }^{3}$, C. Abad ${ }^{4}$, A. Bongiovanni ${ }^{4}$, G. Bruzual ${ }^{4}$, F. Della Prugna ${ }^{4}$, D. Herrera ${ }^{4}$, G. \\ Magris ${ }^{4}$, J. Mateu ${ }^{4}$, R. Pacheco ${ }^{4}$, Ge. Sánchez ${ }^{4}$, Gu. Sánchez ${ }^{4}$, H. Schenner ${ }^{4}$, J. Stock ${ }^{4}$, K. \\ Vieira ${ }^{4}$, F. Fuenmayor ${ }^{1}$, J. Hernandez ${ }^{1}$, O. Naranjo ${ }^{1}$, P. Rosenzweig ${ }^{1}$, C. Secco ${ }^{1}$, G. Spavieri \\ ${ }^{1}$, M. Gebhard ${ }^{5}$, H. Honeycutt ${ }^{5}$, S. Mufson ${ }^{5}$, J. Musser ${ }^{5}$, S. Pravdo ${ }^{10}$, E. Helin ${ }^{10}$, K. \\ Lawrence ${ }^{10}$
}

\begin{abstract}
We describe the discovery circumstances and photometric properties of 2000 EB173, now one of the brightest trans-Neptunian objects (TNOs) with opposition magnitude $m_{R}=18.9$ and also one of the largest Plutinos, found with the drift-scanning camera of the QUEST Collaboration, attached to the 1-m Schmidt telescope of the National Observatory of Venezuela. We measure $B-V=0.99 \pm 0.14$ and $V-R=0.57 \pm 0.05$, a red color observed for many fainter TNOs. At our magnitude limit $m_{R}=20.1 \pm 0.20$, our single detection reveals a sky density of $0.015(+0.034,-0.012)$ TNOs per $\operatorname{deg}^{2}$ (the error bars are $68 \%$ confidence limits), consistent with fainter surveys showing a cumulative number proportional to $10^{0.5 m_{R}}$. Assuming an inclination distribution of TNOs with FWHM exceeding $30 \mathrm{deg}$, it is likely that one hundred to several hundred objects brighter than $m_{R}=20.1$ remain to be discovered.
\end{abstract}

\footnotetext{
${ }^{1}$ Universidad de los Andes, Departamento de Fisica, 5101, Mérida, Venezuela, ferrin@ciens.ula.ve

${ }^{2}$ Yale University, Physics Department, P. O. Box 208121, New Haven CT 06520-8121

${ }^{3}$ Yale University, Astronomy Department, P. O. Box 208101, New Haven CT 06520-8101

${ }^{4}$ Centro de Investigaciones de Astronomía (CIDA), A. P. 264, 5101-A, Mérida, Venezuela

${ }^{5}$ Indiana University, Deptartment of Astronomy, 319 Swain West, Bloomington IN 47405

${ }^{6}$ Ohio State University, Department of Astronomy, Columbus OH 43210

${ }^{7}$ Carnegie Observatories, 813 Santa Barbara St., Pasadena CA 91101

${ }^{8}$ Universidad de Zaragoza, Grupo de Mecanica Espacial, Zaragoza, Spain 50009

${ }^{9}$ Institute of Astronomy, Madingley Road, Cambridge, CB3 OHA, England

${ }^{10}$ Jet Propulsion Laboratory, California Institute of Technology, Pasadena CA 91109
} 
Subject headings: surveys — Kuiper Belt — planets and satellites: individual (2000 EB173)

\section{Introduction}

In recent years, the existence of a belt of small planets with orbits beyond Neptune has been firmly established following the discovery of 1992 QB1 (Jewitt \& Luu 1993). These bodies are believed to be icy planetesimals remaining from the time preceding planet formation, as predicted by Edgeworth (1949) and Kuiper (1951). As of mid-2000, over 300 trans-Neptunian objects (TNOs) are known (Marsden 2000), most having faint magnitudes $\left(20<m_{R}<28\right)$ owing to their detection in narrow-field surveys with large-aperture telescopes. Physical studies have been limited to broadband filter photometry for the fainter bodies and spectroscopy for a few of the brighter objects. These studies show that TNOs are spectrally diverse, with colors ranging from neutral to extremely red (Luu \& Jewitt 1996; Tegler \& Romanishin 1998; Jewitt \& Luu 1998; Noll, Luu, \& Gilmore 2000; Barucci et al. 2000) and with absorption features indicative of hydrocarbons and water ice (Brown et al. 1997; Brown, Cruikshank, \& Pendleton 1999). Previous analyses of the discovery data have shown that the number brighter than $m_{R}$ increases as $10^{\alpha m_{R}}$, with $\alpha$ ranging from $0.52 \pm 0.02$ to 0.76 \pm 0.10 (Jewitt, Luu \& Trujillo 1998; Gladman et al. 1998; Chiang \& Brown 1999) depending on the choice and interpretation of discovery data. It is not known if this luminosity function extends to magnitudes as bright as Pluto $\left(m_{V}=14\right)$ or instead cuts off at intermediate magnitudes. Such a cutoff might reveal a termination in the growth phase of the TNOs at the time of Neptune's formation (Bailey 1993; Stern \& Colwell 1997).

Here we describe the discovery circumstances and photometric properties of 2000 EB173, now one of the brightest TNOs with opposition magnitude $m_{R}=18.9$ and also one of the largest Plutinos (a TNO with a stable orbit like Pluto completing two orbits for every three by Neptune, thereby

avoiding close encounters with Neptune). We show that this detection would not be likely unless the luminosity function continues with a shallow slope $(\alpha \sim 0.5)$ at bright magnitudes, $m_{R}<19$. We also show that the object's photometric properties are similar to those of fainter TNOs with red color.

\section{Observations}

We discovered 2000 EB173 in a computer-aided search through digital images, all recorded in a single 6-hour period on the night of 2000 March 15, using the 1-m Schmidt telescope at Llano del Hato, Venezuela. Our collaboration, known as the Quasar Equatorial Survey Team (QUEST), has designed and constructed for this telescope a digital camera consisting of a $4 \times 4$ mosaic of charge-coupled devices (CCDs), each with 2048 x 2048 15-micron pixels (Snyder 1998). 
The system is designed for drift-scanning. With the telescope position fixed, we align the parallel clocking direction of the CCDs with the East-West motion of the drifting sky image. By clocking each CCD to match the drift rate, we continuously scan 16 separate images. Each scan is 0.588 deg wide in Declination (Dec) and a variable width in Right Ascension (RA) that depends on the duration of the scan (up to $165 \mathrm{deg}$ on long clear nights). Because the columns in our $4 \mathrm{x} 4$ mosaic run East-West, each column yields 4 scans of the same sky area separated in time by 3.8 minutes (the drift time between CCDs). By providing each North-South row in the mosaic with a separate wide-band filter (Johnson U, B, V, or R), each CCD in a column scans the sky through a different band-pass.

Normally, we use this instrument to search for quasars, supernovae, and other variable objects by repeatedly scanning the same area on a nightly basis. To conduct our TNO search, however, we limited the search to an area $28.3 \mathrm{deg}$ wide in RA (centered at RA $=13.249$ hours, Dec $=$ $-1.08 \mathrm{deg}$ ), and scanned the area twice in one night with a separation of 3.99 hours. The total area covered was $66.8 \mathrm{deg}^{2}$, imaged four times per scan through the filter sequence V, R1, B, R2. Later, we processed the data by co-adding the two $\mathrm{R}$ and one $\mathrm{V}$ image from each scan sequence. We then subtracted a scaled reference image created from co-added scans of the same area recorded on previous nights. This removed images of stationary and non-varying stars and galaxies, leaving only images of transient objects, asteroids, and noise artifacts.

We then used a computer program to identify objects with integrated intensities in $\mathrm{R} 1+\mathrm{R} 2+\mathrm{V}$ greater than 3.0 times the noise in the sky background. By combining the set of objects identified in both scan sequences and displaying their images on a computer monitor, possible TNOs appeared as pairs of images. The following constraints were then applied to each object pair: to show retrograde motion; to move within \pm 25 deg of the ecliptic; to have a separation of 5 to 20 arcseconds (the expected displacement in 3.99 hours of a TNO observed near opposition); to reveal the same approximate magnitude in the 3.99-hour interval; to exhibit a FWHM consistent with surrounding stars; and to be detected in the separate R1, R2, and V images before co-addition (6 independent observations). The only candidate (out of 600,000 ) to meet all these requirements was 2000 EB173.

\section{Results}

Efficiency. We estimated the limiting magnitude of our search by identifying main-belt asteroids appearing in a single scan sequence taken near opposition on an earlier night through the same filter set. Given the 7.6-minute interval between successive R exposures in a single scan, the near-opposition motion of main-belt asteroids is 4 to 6 pixels. We could therefore use the detection procedure described above, but with the two $R$ images replacing each of the two co-added $R+R+V$ images from the TNO scans.

Figure 1 shows the number of main-belt asteroids we detect as a function of $m_{R}$. Our relative detection efficiency is the observed distribution divided by a power-law fit at bright magnitudes 
(Rabinowitz 1994). As shown in the figure, our $50 \%$ detection limit occurs at $m_{R}=19.6 \pm 0.2$. We also determined that our absolute detection efficiency at bright magnitudes is close to $100 \%$ by verifying incidental detections of catalogued asteroids within the search fields.

We expect that our detection efficiency for TNOs has the same form as for main-belt asteroids, but shifted to fainter magnitudes because we co-add two $\mathrm{R}$ images and one $\mathrm{V}$ image for each TNO detection. This increases the relative signal from each TNO and from the sky by a factor of $2.6 \pm 0.1$, as verified from our measurements of 2000 EB173. The signal to noise ratio increases by the square root of this factor since the noise at the detection limit is dominated by counting statistics of the sky signal. Because we searched for TNOs and main-belt asteroids with the same threshold value of the signal to noise ratio, the limiting magnitude for our TNO search is fainter by a factor $2.5 \log (\sqrt{2.6 \pm 0.1})$, yielding magnitude limit $m_{R}=20.1 \pm 0.2$.

Orbit. Following the discovery of 2000 EB173, we confirmed its motion in QUEST scans from previous nights. Based on an orbit solution from these initial observations we recovered the object three months later with the 1-m Yale telescope at Cerro Tololo, Chile. Table 1 shows the most recent orbital elements determined for 2000 EB173 from observations spanning 4 years (Marsden and Williams 2000a). These include pre-discovery, photographic observations with the 48" Schmidt telescope at Palomar, and post-discovery observations by amateur astronomers with telescopes of sub-meter aperture (Marsden and Williams 2000b). The eccentricity, $e$, and inclination, $i$, are typical values for a Plutino (Jewitt \& Luu 1996), while the semimajor axis, $a$, is relatively low. Given the long arc of the observations, the orbit solution is accurate to better than $1 \%$.

Size. The absolute magnitude we determine, $H=4.7 \pm 0.1$, is one of the brightest for any known TNO, making 2000 EB173 also one of the largest TNOs assuming all have similar albedos. The only brighter TNO is 1996 TO66 with $H=4.5$ and the next brightest is 1999 TC36, a Plutino with $H=4.8$ (Marsden 2000). There are no known geometric albedos for TNOs, so one has to be assumed. For an albedo of 0.04, typical of dark, carbon-rich asteroids and cometary nuclei and a value commonly assumed for TNOs, the corresponding diameter would be $\sim 600 \mathrm{~km}$, about $1 / 4$ the size of Pluto (Jewitt, Luu \& Trujillo 1998) and marginally resolvable with the Hubble Space Telescope.

Cumulative number. Figure 2 shows the number of TNOs per $\operatorname{deg}^{2}$ we find brighter than our detection limit, plotted together with previous measurements at mostly fainter limits. Also shown are two previously suggested curves to fit the observations, $\alpha=0.58 \pm 0.05$ from Jewitt, Luu \& Trujillo (1998) and $\alpha=0.76 \pm 0.1$ from Gladman et al. (1998). Our detection of 1 TNO per 66.8 $\operatorname{deg}^{2}$ favors the $\alpha=0.58$ curve. It also rules out the previously established upper limit (Kowal 1989) of 1 TNO per $1000 \mathrm{deg}^{2}$ brighter than $m_{R}=19.5$, as this would require an unrealistic, sharp cutoff in the magnitude frequency above $m_{R}=20.1$. Given previously predicted magnitude-frequency curves that assume an upper limit to the diameter distribution of TNOs (Jewitt, Luu, \& Trujillo 1998), the likelihood of our discovery would be less than $10 \%$ for any diameter cutoff below 500 $\mathrm{km}$. 
Photometry and imaging. Following our discovery of 2000 EB173 we made photometric R-band observations with the 1-m Yale telescope, BVRI observations with the 2.4-m Michigan-DartmouthMIT (MDM) telescope at Kitt Peak, and V and R observations with the 3.5-m Wisconsin-IndianaYale-NOAO (WIYN) telescope, also at Kitt Peak. Incidental, unfiltered CCD observations were also made by the Near Earth Asteroid Tracking Program (NEAT) of the Jet Propulsion Laboratory using a U.S. Air Force 1.2-m telescope at Haleakala, Maui (these observations were identified using the SkyMorph archive at http://skys.gsfc.nasa.gov/skymorph/skymorph.html).

Table 2 lists the results from these observations and from pre-discovery QUEST scans. From Mar 1 to Mar 15, and from June 3 to June 15, no variability in $m_{R}$ greater than $10 \%$ appears. Furthermore, a series of 9 images in V taken June 15 shows no variability greater than $3 \%$ over a 1.25-hour period. Co-addition of the June 15 images reveals a stellar point-spread function for 2000 EB173, with any cometary activity contributing less than $10 \%$ to the flux outside a radius of 0.6 arcseconds. We are therefore confident that the colors we measure are unaffected by intrinsic variability from rotation or cometary outbursts.

Colors. Figure 3 shows the spectral reflectance we derive for 2000 EB173 along with the measured reflectance for other TNOs (Jewitt \& Luu 1998). Shortwards of 0.7 microns, the spectral slope we observe is consistent with that of other other TNOs. This result places the object among a group with red colors identified previously by Tegler \& Romanishin (1998). Longwards of 0.7 microns, we observe a possible transition to a neutral reflectance, but this result is ambiguous. Depending on the pixel aperture we use for our photometry, we also obtain a red $R-I$ color for 2000 EB173, which would be expected based on previous observations of fainter red TNOs. In only a few cases has such a flattening of the reflectance been observed (e.g., $1993 \mathrm{FW}$ as observed by Barucci et al. 2000).

\section{Conclusions}

1. The unusual brightness of 2000 EB173 provides an opportunity to study the physical nature of one TNO with substantially greater precision than has been possible previously. It now becomes possible for all astronomers, including those with modest facilities, to make a valuable contribution to the study of the primordial bodies preserved in the outer solar system. Possible contributions would be to determine the size of 2000 EB173 by measuring the albedo with infrared observations or by direct observation with space-based telescopes. Such studies would have important implications for the total mass of the TNO population, currently uncertain by more than an order of magnitude.

2. We observe no variability in the lightcurve of 2000 EB173 and no cometary coma. The color is very red shortwards of 0.7 microns, typical of many fainter TNOs. Our measurements indicate a possible flattening of the reflectance longwards of 0.7 microns, but are also consistent with a redder color.

3. Our detection of one TNO brighter than $m_{R}=20.1$ in a search of $66.8 \mathrm{deg}^{2}$ favors the 
flat luminosity function $(\alpha=0.58)$ of Jewitt, Luu, \& Trujillo (1998) over the steeper distribution ( $\alpha=0.768)$ of Gladman et al. (1998).

4. Extrapolating to a search of the entire area within $60 \mathrm{deg}$ of the ecliptic (within the inclination range for TNOs determined by Jewitt, Luu, \& Chen 1996), one could reasonably expect to find from one hundred to several hundred TNOs brighter than $m_{R}=20.1$. A dedicated search using a telescope with capabilities similar to our own would discover most of these bodies in a few years.

This work was supported by the National Science Foundation, the Department of Energy, and

the National Aeronautics and Space Administration. The Observatorio Astronómico Nacional is operated by CIDA for the Consejo Nacional de Investigaciones Científicas y Tecnológicas. The Council for Scientific and Technological Developmentof the University of the Andes also provided support.

\section{REFERENCES}

Bailey, M.E., in IAU Symp. 160, Asteroids, Comets, Meteors 1993, ed. A. Milani, N. Di Martino, \& A. Cellino (Dordrecht, Kluwer), 443

Barucci, M.A., Romon, J., Doressoundiram, A., \& Tholen, D.J., 2000, AJ, 120, 496.

Brown, R.H., Cruikshank, D.P., Pendleton, Y., \& Veeder, G.J., 1997, Science, 276, 937.

Brown, R.H., Cruikshank, D.P., \& Pendleton, Y., 1999, ApJ, 519, L101.

Chiang, E.I., \& Brown, M.E., 1999, AJ, 118, 1411

Cochran, A.L., Levison, H.F., Tamblyn, P., Stern, A.S., \& Duncan, M.J., 1998, AJ, 503, L89

Edgeworth, K. E., 1949, MNRAS, 109, 600

Gladman, B., Kavelaars, J.J., Nicholson, P.D., Loredo, T.J., \& Burns, J.A., 1998, AJ, 116, 2042

Irwin, M., Tremaine, S., \& Zytkow, A., 1995, AJ, 110, 3082

Jewitt, D., \& Luu, J., 1993, Nature, 362, 730

Jewitt, D., Luu, J., \& Chen, J., 1996, AJ, 112, 1225

Jewitt, D., \& Luu, J., 1996, in Completing the Inventory of the Solar System, ed. T. Rettig \& J. Hahn (California, Astronomical Society of the Pacific), 255

Jewitt, D., Luu, J., \& Trujillo, C., 1998, AJ, 115, 2125

Jewitt, D., \& Luu, J., 1998, AJ, 115, 1667 
Kowal, C.T., 1989, Icarus, 77, 118

Kuiper, G.P., in Astrophysics, ed. J. Hynek (New York: McGraw-Hill), 357

Levison, H., \& Duncan, M., 1990, AJ, 100, 1669

Luu, J., \& Jewitt, D., 1988, AJ, 951256

Luu, J., \& Jewitt, D., 1996, AJ, 112, 2310

Luu, J., \& Jewitt, D., 1998, ApJ, 502, L91

Marsden, B.G., 2000, List of Transneptunian objects. Internet web page for the International Astronomical Union: Minor Planet Center (http://cfa-www.harvard.edu/iau/lists/TNOs.html), referenced Nov. 26, 2000

Marsden, B.G., \& Williams, G.V., 2000a, Minor Planet Electronic Circular 2000-V38

Marsden, B.G., \& Williams, G.V., 2000b, Minor Planet Electronic Circular 40875, 40981-40984

Noll, K.S., Luu, J., \& Gilmore, D., 2000, AJ, 119, 970.

Rabinowitz, D.L., 1994, Icarus, 111, 364.

Sheppard, S.S., Jewitt, D.D., Trujillo, C.A., Brown, M.J., Ashley, M.C., 2000, AJ, in press.

Snyder, J. A., 1998, SPIE, 3355, 635

Stern, S., \& Colwell, J.E., 1997, AJ, 114, 841

Tegler, S.C., \& Romanishin, W., 1998, Nature, 392, 49

Tombaugh, C., 1961, in Planets and Satellites, ed. G.P. Kuiper \& B. M. Middlehurst (Chicago: Univ. Chicago Press), 12

Correspondence and requests for materials should be addressed to David Rabinowitz, Yale University Physics Dept., P. O. Box 208121, New Haven CT 06520-8121; or by E-mail to david.rabinowitz@yale.edu. 
Table 1. Orbital Elements for 2000 EB173

\begin{tabular}{cccccccc}
\hline \hline$a(\mathrm{AU})$ & $e$ & $i\left(^{\circ}\right)$ & $w\left(^{\circ}\right)$ & $\operatorname{Node}\left(^{\circ}\right)$ & $M\left(^{\circ}\right)$ & $H$ & Epoch \\
\hline 39.003 & 0.266 & 15.493 & 66.981 & 169.359 & 339.028 & $4.7 \pm 0.1$ & 2451800.5 \\
\hline
\end{tabular}

Note. - Orbital Elements are J2000 from Marsden \& Williams (2000a) with uncertainties less than $1 \%$. The value for $H$ derives from the $\mathrm{R}$ magnitude, $m_{R}=18.90 \pm 0.10$, observed at the lowest phase angle, $\alpha=0.29^{\circ}$, on 2000 April 11 UT (see Table 2) and from the mean $V-R$ color $(0.574 \pm 0.047)$ determined from observations at all phase angles. 
Table 2. Photometric Observations of 2000 EB173

\begin{tabular}{|c|c|c|c|c|c|c|c|c|c|}
\hline Date(2000) & $B$ & $V$ & $R$ & $I$ & $B-V$ & $V-R$ & $V-I$ & $a\left(^{\circ}\right)$ & Telescope \\
\hline Feb 11.30 & $\ldots$ & 20.01 & 19.32 & $\ldots$ & $\ldots$ & 0.69 & $\ldots$ & 1.60 & 1-m Schmidt \\
\hline$\cdots$ & $\ldots$ & \pm 0.17 & \pm 0.06 & $\cdots$ & $\cdots$ & \pm 0.18 & $\cdots$ & $\cdots$ & $\cdots$ \\
\hline Feb 12.30 & $\ldots$ & 19.78 & 19.34 & $\ldots$ & $\ldots$ & 0.44 & $\ldots$ & 1.59 & 1-m Schmidt \\
\hline$\ldots$ & $\ldots$ & \pm 0.15 & \pm 0.06 & $\ldots$ & $\ldots$ & \pm 0.16 & $\ldots$ & $\ldots$ & $\ldots$ \\
\hline Feb 13.30 & $\cdots$ & $\cdots$ & 19.27 & $\cdots$ & $\cdots$ & $\cdots$ & $\cdots$ & 1.57 & 1-m Schmidt \\
\hline$\cdots$ & $\cdots$ & $\cdots$ & \pm 0.05 & $\cdots$ & $\cdots$ & $\cdots$ & $\cdots$ & $\cdots$ & \\
\hline Feb 14.30 & $\ldots$ & $\ldots$ & 19.51 & $\ldots$ & $\ldots$ & $\ldots$ & $\ldots$ & 1.55 & 1-m Schmidt \\
\hline$\cdots$ & $\cdots$ & $\cdots$ & \pm 0.10 & $\cdots$ & $\cdots$ & $\cdots$ & $\cdots$ & $\cdots$ & $\cdots$ \\
\hline Mar 01.34 & $\ldots$ & 19.84 & 19.30 & $\ldots$ & $\ldots$ & 0.54 & $\ldots$ & 1.19 & 1-m Schmidt \\
\hline$\cdots$ & $\ldots$ & \pm 0.14 & \pm 0.05 & $\cdots$ & $\cdots$ & \pm 0.15 & $\cdots$ & $\cdots$ & $\cdots$ \\
\hline Mar 03.33 & $\ldots$ & 19.99 & 19.32 & $\cdots$ & $\cdots$ & 0.67 & $\ldots$ & 1.14 & 1-m Schmidt \\
\hline$\cdots$ & $\ldots$ & \pm 0.17 & \pm 0.06 & $\cdots$ & $\cdots$ & \pm 0.18 & $\cdots$ & $\cdots$ & $\cdots$ \\
\hline Mar 08.28 & $\cdots$ & 20.03 & 19.27 & $\cdots$ & $\cdots$ & 0.76 & $\cdots$ & 1.01 & 1-m Schmidt \\
\hline$\ldots$ & $\ldots$ & \pm 0.15 & \pm 0.05 & $\ldots$ & $\ldots$ & \pm 0.16 & $\ldots$ & $\ldots$ & $\ldots$ \\
\hline Mar 10.30 & $\cdots$ & 19.79 & 19.39 & $\cdots$ & $\cdots$ & 0.40 & $\cdots$ & 0.95 & 1-m Schmidt \\
\hline$\cdots$ & $\cdots$ & \pm 0.16 & \pm 0.08 & $\ldots$ & $\cdots$ & \pm 0.18 & $\cdots$ & $\cdots$ & $\cdots$ \\
\hline Mar 11.28 & $\ldots$ & 19.80 & 19.19 & $\ldots$ & $\ldots$ & 0.61 & $\ldots$ & 0.92 & 1-m Schmidt \\
\hline$\ldots$ & $\ldots$ & \pm 0.14 & \pm 0.06 & $\ldots$ & $\ldots$ & \pm 0.015 & $\ldots$ & $\ldots$ & $\ldots$ \\
\hline Mar 15.19 & $\cdots$ & 19.65 & 19.31 & $\cdots$ & $\cdots$ & 0.34 & $\cdots$ & 0.81 & 1-m Schmidt \\
\hline$\ldots$ & $\cdots$ & \pm 0.16 & \pm 0.08 & $\cdots$ & $\cdots$ & \pm 0.18 & $\cdots$ & $\cdots$ & $\cdots$ \\
\hline Mar 15.35 & $\cdots$ & 19.94 & 19.21 & $\cdots$ & $\cdots$ & 0.73 & $\cdots$ & 0.81 & 1-m Schmidt \\
\hline$\cdots$ & $\cdots$ & \pm 0.15 & \pm 0.05 & $\cdots$ & $\cdots$ & \pm 0.16 & $\cdots$ & $\cdots$ & $\cdots$ \\
\hline Apr 11.47 & $\cdots$ & $\cdots$ & 18.90 & $\cdots$ & $\cdots$ & $\cdots$ & $\cdots$ & 0.29 & NEAT $1.2-\mathrm{m}$ \\
\hline$\ldots$ & $\ldots$ & $\ldots$ & \pm 0.10 & $\ldots$ & $\ldots$ & $\cdots$ & $\ldots$ & $\ldots$ & $\ldots$ \\
\hline Jun 03.99 & $\ldots$ & $\ldots$ & 19.40 & $\ldots$ & $\ldots$ & $\ldots$ & $\ldots$ & 1.62 & Yale 1-m \\
\hline$\cdots$ & $\cdots$ & $\ldots$ & \pm 0.12 & $\cdots$ & $\cdots$ & $\cdots$ & $\cdots$ & $\cdots$ & $\ldots$ \\
\hline Jun 10.16 & 21.10 & 20.11 & 19.51 & 19.13 & 0.99 & 0.60 & 0.98 & 1.73 & MDM 2.4-m \\
\hline$\ldots$ & \pm 0.11 & \pm 0.08 & \pm 0.06 & $\pm 0.07^{\mathrm{a}}$ & \pm 0.14 & \pm 0.10 & $\pm 0.11^{\mathrm{a}}$ & $\ldots$ & \\
\hline Jun 15.20 & $\cdots$ & 20.18 & 19.63 & $\cdots$ & $\cdots$ & 0.55 & $\cdots$ & 1.80 & WIYN 3.5-m \\
\hline$\ldots$ & $\ldots$ & \pm 0.03 & \pm 0.03 & $\ldots$ & $\ldots$ & \pm 0.05 & $\ldots$ & $\ldots$ & $\cdots$ \\
\hline
\end{tabular}

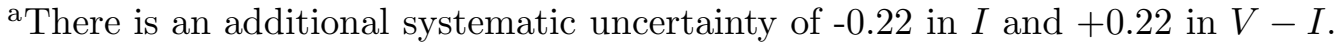

Note. - A common set of calibrated field stars were photometric standards for the 1-m Schmidt observations. For the Yale and WIYN observations, the photometric standard was a single field star calibrated against catalogue standards with MDM. The NEAT observations were CCD observations with no filter, calibrated against secondary photometric standards observed in $\mathrm{V}$ an $\mathrm{R}$ with the 1-m Schmidt (a color correction term was determined assuming the mean $V-R$ color, $0.574 \pm 0.047$, observed for 2000 EB173 on other nights). All colors are Kron-Cousins. 


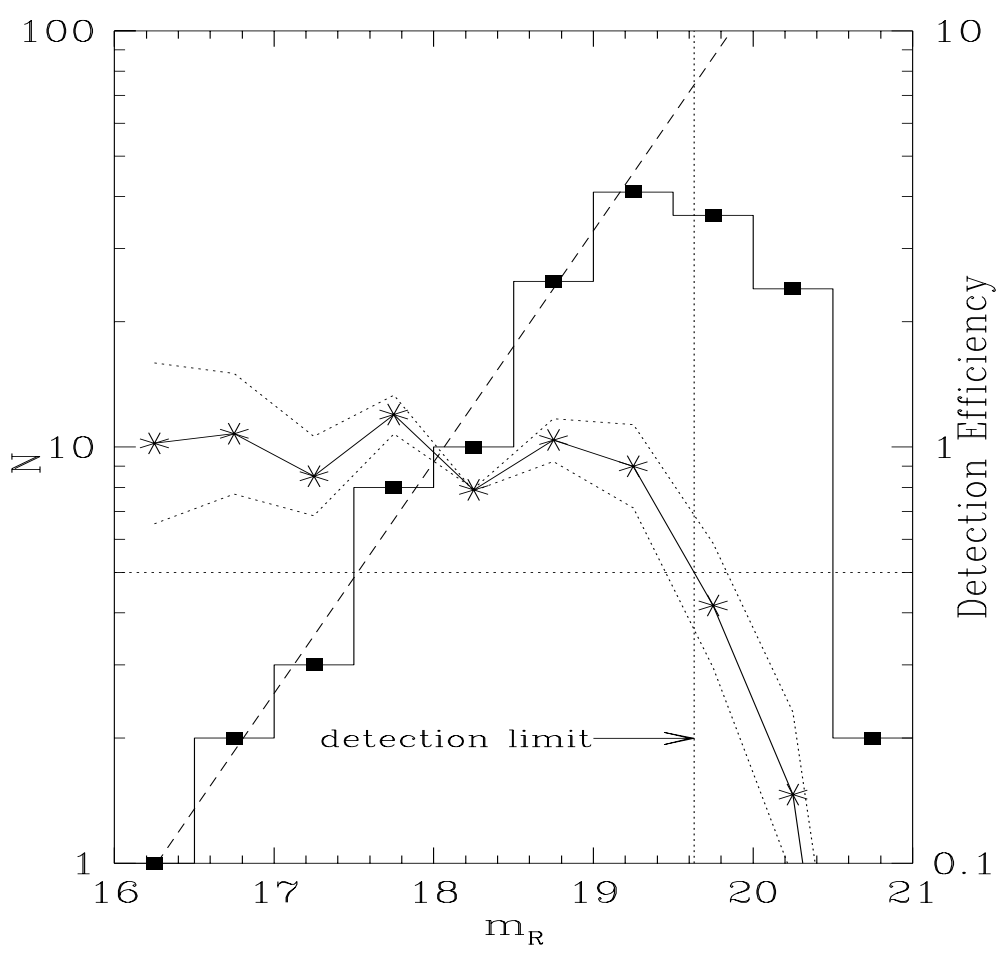

Fig. 1.- The number of detected main-belt asteroids, $N$ (squares), and the detection efficiency (stars) vs apparent $\mathrm{R}$ magnitude, $m_{R}$. The detection efficiency is $N$ divided by a power-law fit to $N$ vs $m_{R}$ for $m_{R}<19.0$ (dashed line). The dotted curves show the uncertainty in the efficiency owing to the uncertainty in the fit. The dotted lines show the $50 \%$ detection limit at $m_{R}=19.63 \pm 0.20$. For TNOs, the detection limit is fainter by 0.51 magnitudes. 


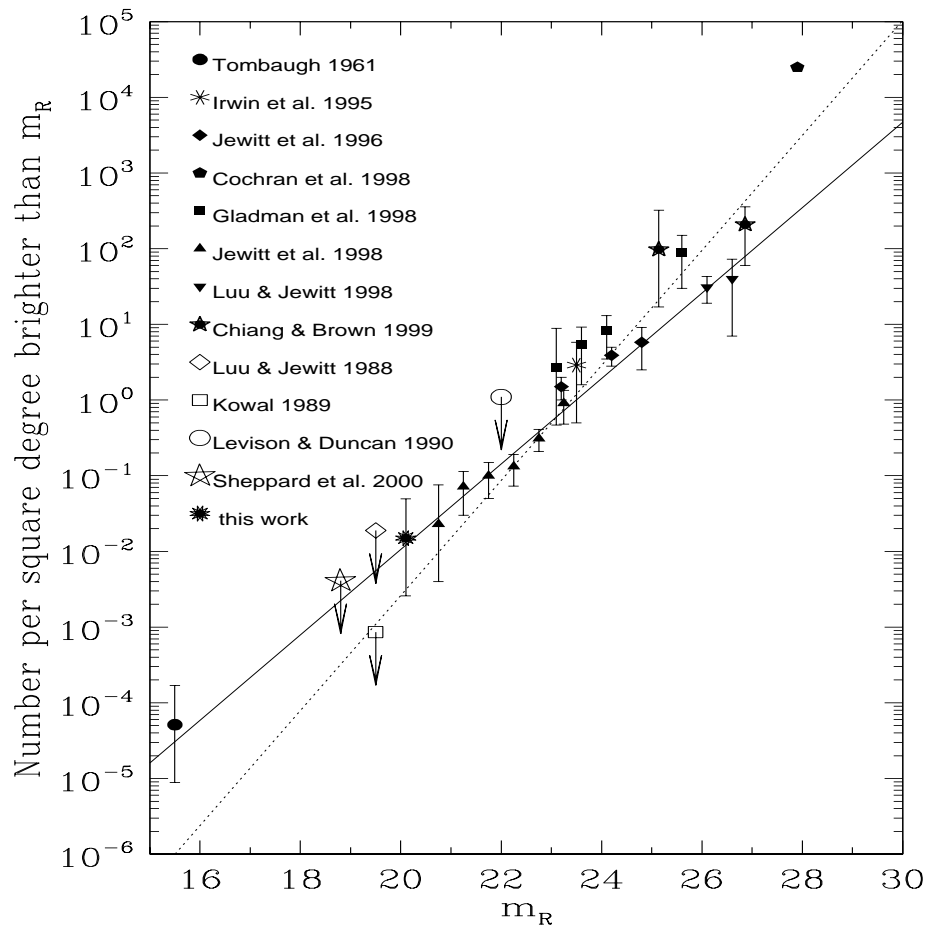

Fig. 2. - The cumulative number of TNOs per $\operatorname{deg}^{2}$ brighter than magnitude limit $m_{R}$ from this paper and from previous surveys. Where the number per $\operatorname{deg}^{2}$ is based on a single detection, we assign error bars corresponding to a $68 \%$ confidence interval (i.e., the lowest and highest number per $\operatorname{deg}^{2}, n$, for which the likelihood of detecting at least and at most one TNO, respectively, exceeds $16 \%$. For a given search area, $A$, this corresponds to the respective values of $n$ for which the summations from $k=1$ to $\infty$ and from $k=0$ to 1 of the Poisson likelihood $e^{-n A}(n A)^{k} / k$ ! equal 0.16). Where the survey limit is reported as a V magnitude (Luu \& Jewitt 1998, Kowal 1989, Cochran et al. 1998), we assume $V-R=0.5$. The solid and dashed lines are two previously derived fits (Jewitt, Luu, \& Trujillo 1998; Gladman et al. 1998) that assume a magnitude frequency proportional to $10^{\alpha m_{R}}$ with $\alpha=0.58 \pm 0.05$ and $\alpha=0.76 \pm 0.11$, respectively. 


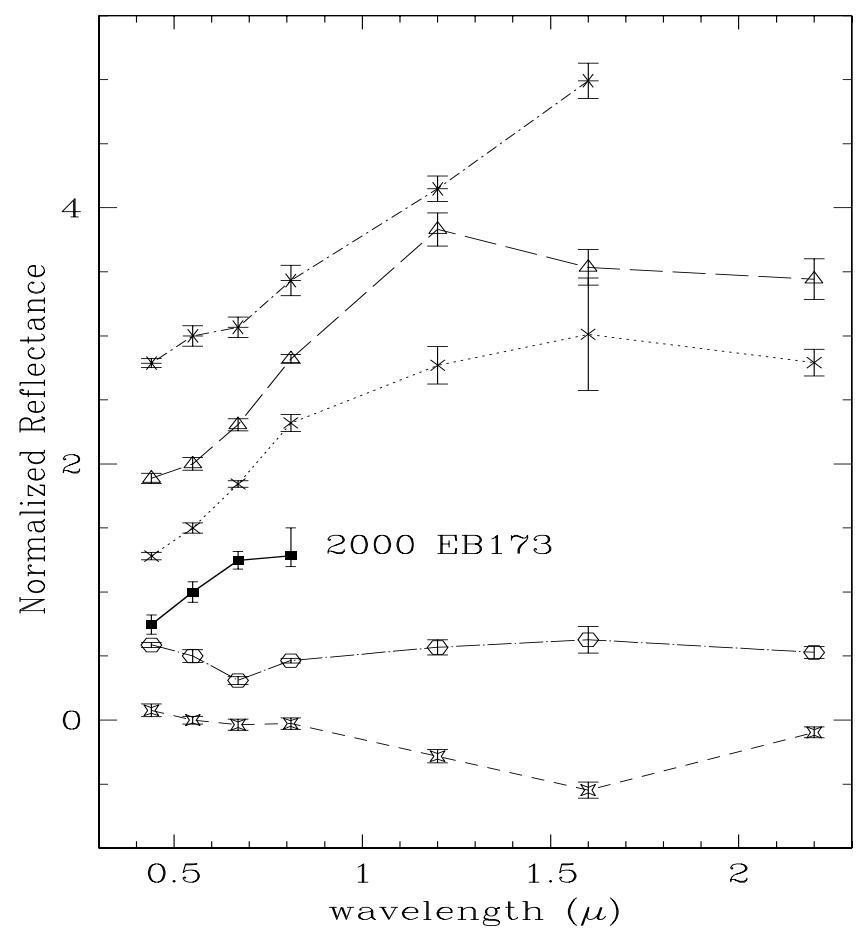

Fig. 3.- Spectral reflectance for 2000 EB173 (filled squares) and for previously observed TNOs (from top to bottom: 1996 TS66, 1996 TP66, 1993 SC, 1996 TL66, and 1996 TO66) assuming solar colors $(B-V, V-R$, and $V-I)$ given by Jewitt \& Luu (1998). All spectra are normalized to unity at 0.55 microns. They have been shifted vertically, but share the same scale. 